\title{
Recurrent branch of anterior interosseous artery perforator-based propeller flap for distal forearm injuries: Report of 2 cases
}

\author{
Ryusho Hamada MD ${ }^{1,2}$ ( ) Akira Shinaoka MD, $\mathrm{PhD}^{2,3}$ | Narushi Sugiyama MD, $\mathrm{PhD}^{2}$ | \\ Toshiyuki Watanabe MD $^{2}$ | Yuki Miura MD ${ }^{1,2}$ | Yoshihiro Kimata MD, PhD ${ }^{2}$
}

${ }^{1}$ Department of Plastic Surgery, Kagawa Prefectural Central Hospital, Kagawa, Japan

${ }^{2}$ Department of Plastic and Reconstructive Surgery, Okayama University Graduate School of Medicine, Dentistry, and Pharmaceutical Science, Okayama, Japan

${ }^{3}$ Department of Human Morphology, Okayama University Graduate School of Medicine, Dentistry, and Pharmaceutical Science, Okayama, Japan

Correspondence

Ryusho Hamada, Department of Plastic

Surgery, Kagawa Prefectural Central Hospital

1-2-1 Asahi-cho, Takamatsu-shi, Kagawa

760-8557, Japan.

Email: patapatagonia.r3@gmail.com

\begin{abstract}
Despite various options for the reconstruction of soft tissue defects in the distal forearm, perforator-based propeller flap is rarely used. Here, we presented 2 cases of distal forearm injuries that were repaired using the recurrent branch of anterior interosseous artery perforatorbased propeller flap. Patients in these cases were 57 and 67 years of age. Wounds resulting from farming machine injury and pyogenic extensor tenosynovitis following cat bite wounds were localized to the distal forearm and dorsum of the hand. Defect dimensions were $5 \mathrm{~cm} \times 10$ $\mathrm{cm}$ and $5 \mathrm{~cm} \times 8 \mathrm{~cm}$. The $12 \mathrm{~cm} \times 7 \mathrm{~cm}$ and $21 \mathrm{~cm} \times 4 \mathrm{~cm}$ sized recurrent branch of anterior interosseous artery perforator-based propeller flap was designed adjacent to the wounds. In the latter case, the absence of the posterior interosseous artery in the distal forearm was observed. One perforator from the recurrent branch of the anterior interosseous artery emerged through the septum between the extensor digiti minimi and extensor carpi ulnaris $7.5 \mathrm{~cm}$ and $6.0 \mathrm{~cm}$ proximal to the ulnar head in cases 1 and 2, respectively. Perforators were identified using multidetector computed tomographic angiography and handheld Doppler. Extending to two-thirds or almost the full length of the forearm, the flaps were raised and rotated by $90^{\circ}$ and $120^{\circ}$ to cover the defect. The donor sites were closed using free skin graft. Both flaps survived. Except for minor wound dehiscence and hemarthrosis, no other postoperative complications occurred. Patients returned to work or daily activities at 3- and 4-month follow-up after surgery.
\end{abstract}

\section{1 | INTRODUCTION}

The reconstruction of traumatic soft tissue defects in the distal forearm remains challenging for reconstructive surgeons. The primary restoration of well-vascularized tissue around the exposed vital structures such as the bone, tendons, nerves, and vessels is required to prevent infection and tendon adhesion in the distal forearm (Shaw \& Payne, 1946). The type of flap considered the most efficacious in the management of soft tissue defects in the distal forearm remains controversial. The reverse flap is popular for distal forearm reconstruction (Biemer \& Stock, 1983; Li, Liu, \& Cao, 1989; Zancolli \& Angrigiani, 1986). However, reverse flaps with retrograde radial or ulnar artery pedicle have a disadvantage in that the major vessels of the forearm have to be sacrificed, which could result in postoperative digital ischemia or cold intolerance (Jones \& O'Brien, 1985). The reverse posterior interosseous artery flap can be applied in cases with ulnar or radial artery disruption to cover skin defects over the distal forearm without sacrificing the major vessels. However, it requires the retrograde dissection of the vascular pedicle through the fascial septum, and there is the potential for posterior interosseous nerve injury when raising the flap (Buchler \& Frey, 1991) and postoperative venous congestion (Akinci, Ay, Kamiloglu, \& Erçetin, 2006; Özalp, Elbey, Aydin, \& Özkan, 2016). Moreover, retrospective clinical studies on the posterior interosseous artery flap reported that the posterior interosseous artery was absent or unusable in some cases (Cavadas, 1999; Dadalt Filho, Ulson, \& Penteado, 1994; Giunta \& Lukas, 1998). Perforator-based propeller flaps can provide adequate soft tissue coverage for the distal forearm with antegrade venous drainage without sacrificing the major vessels (Hyakusoku, Yamamoto, \& Fumiiri, 1991). Recently, there have been several reports focusing on the anterior interosseous artery perforator-based flap; however, reports describing perforator-based propeller flap in the distal forearm are rare (Akin, 2003; 
Panse et al., 2017; Panse \& Sahasrabudhe, 2014; Syed et al., 1997; Yii \& Niranjan, 1999). Here, we presented 2 cases of distal forearm defects following a farming machine injury and pyogenic extensor tenosynovitis that were repaired using the recurrent branch of anterior interosseous artery perforator-based propeller flap.

\section{2 | CASE REPORTS}

\section{1 | Case 1}

A 57-year-old male farm worker presented to our clinic after a farming machine injury to his right distal forearm (Figure 1A). The complex injury comprised distal radius fracture, disruption of the radial flexor, near complete disruption of the extensor tendons, segmental loss of the radial artery and superficial branch of the radial nerve, and a soft tissue defect measuring $5 \mathrm{~cm} \times 10 \mathrm{~cm}$ on both the dorsal and radial aspects at the distal forearm level (Figure 1B). The wounds were first treated with meticulous debridement, tendon repair, and vacuumassisted closure therapy by orthopedic surgeons. Open reduction with internal plate fixation for the radius fracture was performed at 4 days after initial debridement (Figure 1C); moreover, the recurrent branch of anterior interosseous artery perforator-based propeller flap was raised to cover the soft tissue defect. A dominant perforator from the recurrent branch of the anterior interosseous artery $7.5 \mathrm{~cm}$ proximal to the ulnar head was detected preoperatively using computed tomographic (CT) angiography and handheld Doppler. The recurrent branch of anterior interosseous artery perforator-based propeller flap was designed adjacent to the wound (Figure 1D). The proximal side of the flap was first raised under the deep fascia up to the muscle tendon junction and above the paratenon distally. A healthy septocutaneous perforator emerged through the septum between the extensor digiti minimi and extensor carpi ulnaris (Figure 1E). After fully elevating the

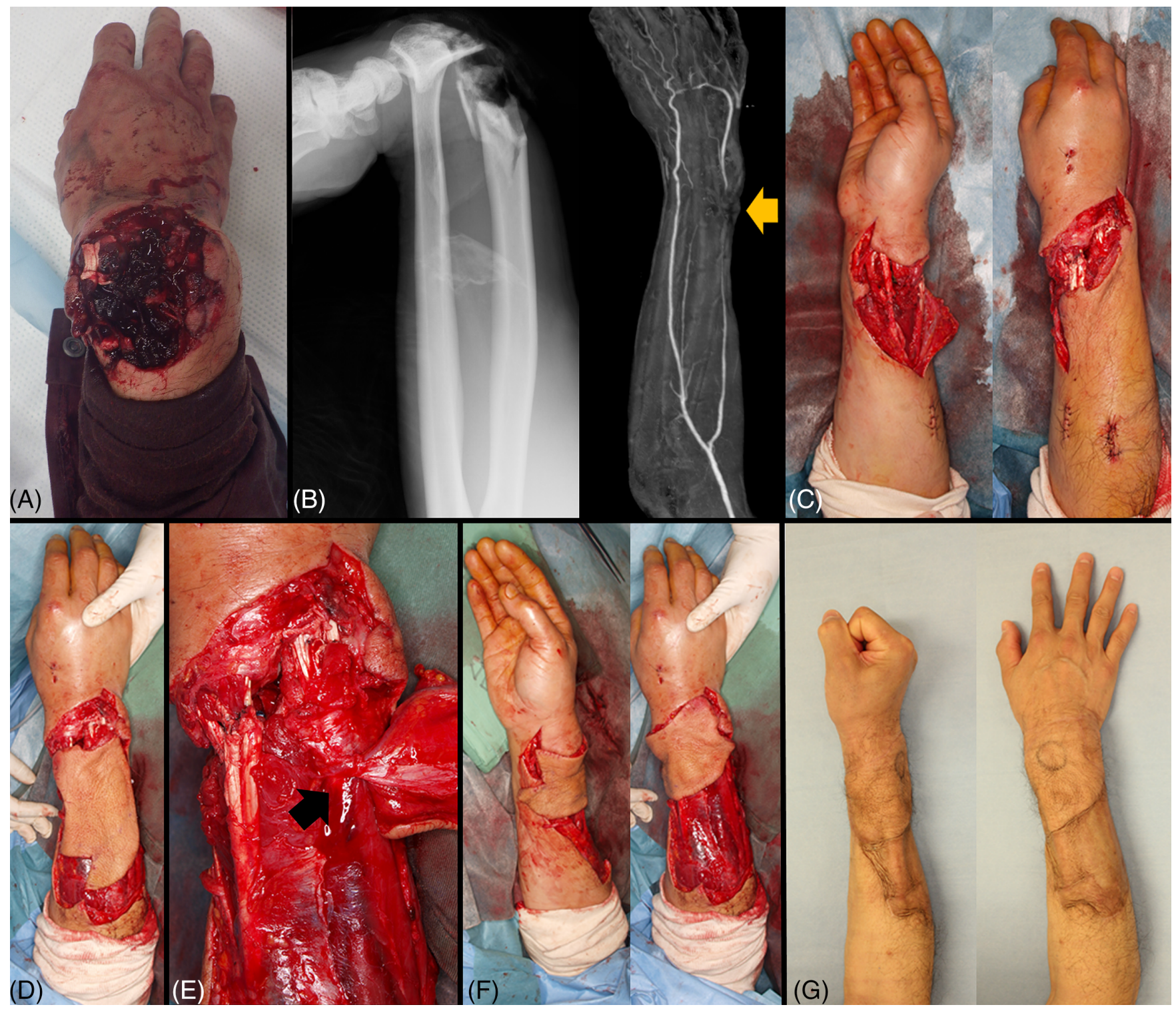

FIGURE 1 A, Wound on the right distal forearm. B, Distal radius fracture and disruption of the radial artery. C, After performance of repeated debridement. D, After flap elevation. E, Recurrent branch of the anterior interosseous artery perforator (black arrow). $F, A f t e r ~ 90^{\circ}$ of rotation. G, At 6 months after surgery 


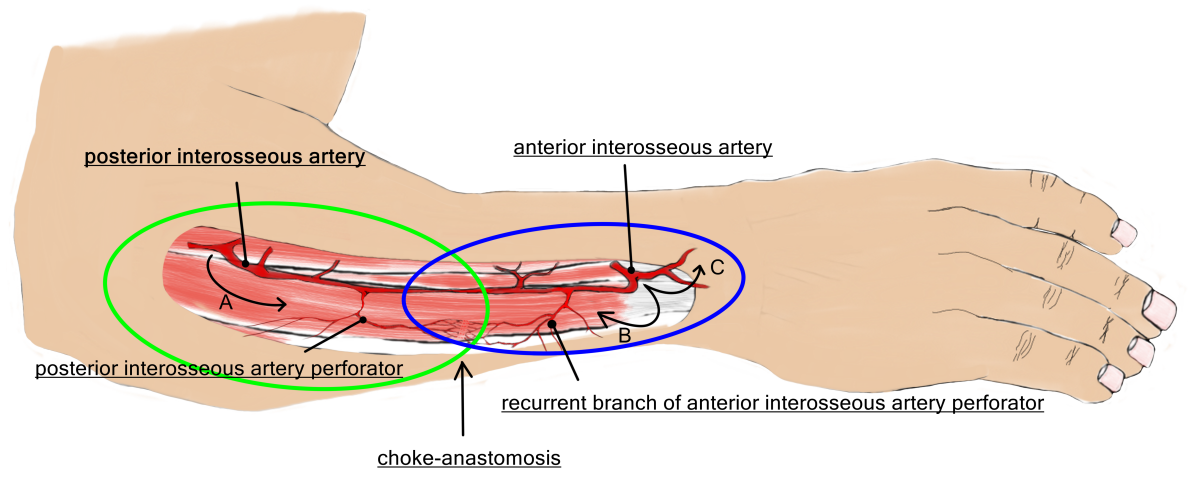

choke-anastomosis

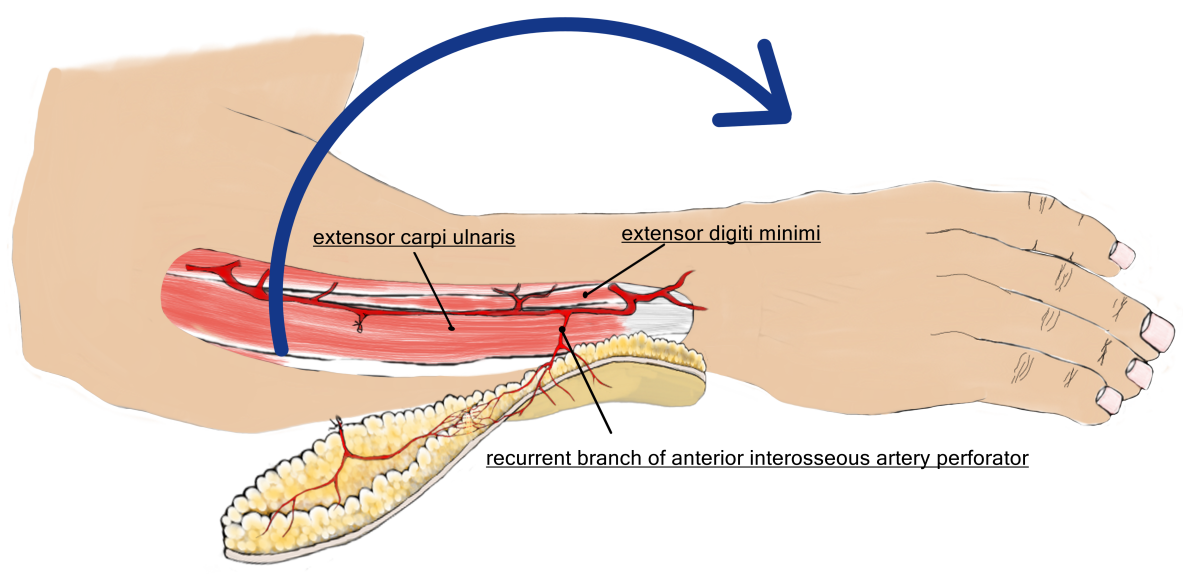

FIGURE 2 Illustration of the recurrent branch of anterior interosseous artery perforator-based propeller flap. A, Posterior interosseous artery. B, Recurrent branch of the anterior interosseous artery between the extensor digiti minimi and extensor carpi ulnaris. C, Dorsal branch of the anterior interosseous artery. The blue circle pertains to the primary vascular territory from the recurrent branch of the anterior interosseous artery, whereas the green circle indicates the adjacent vascular territory from the posterior interosseous artery. The blue arrow is the rotation arc of the recurrent branch of anterior interosseous artery perforator-based propeller flap

flap (Figure 2), it was rotated by $90^{\circ}$ clockwise to cover the defect (Figure 1F). The size of the flap was $12 \mathrm{~cm} \times 7 \mathrm{~cm}$. The donor site was closed using a free skin graft. The flap developed minor wound dehiscence at its distal tip and required resuturing; nevertheless, the wound healed with no other major complications such as venous congestion or infection. The patient was referred to the rehabilitation department at 17 days postinjury and returned to daily activities and work at 3-month follow-up (Figure 1G).

\subsection{Case 2}

A 67-year-old healthy female presented with infected cat bite wounds on the right distal forearm at 10 days postinjury (Figure $3 \mathrm{~A}$ ). Pyogenic extensor tenosynovitis progressed from the middle third of the forearm to the third metacarpophalangeal (MCP) joint, and almost the entire extensor tendons had ruptured or degenerated at the wrist level (Figure 3B). The patient was treated with serial debridement and vacuum-assisted closure therapy by orthopedic surgeons. There was a soft tissue defect measuring $5 \mathrm{~cm} \times 8 \mathrm{~cm}$ in the dorsal hand with exposure of the third MCP joint and ruptured extensor tendons after multiple debridements (Figure $3 \mathrm{C}$ ). In this case, the use of reverse posterior interosseous artery flap was initially planned; however, the flap was not usable owing to the absence of the posterior interosseous artery in the distal forearm, which might be because of anatomic variation or previous surgical intervention. We changed the procedure intraoperatively and adopted a sequential surgical approach based on the recurrent branch of anterior interosseous artery perforator. A dominant perforator from the recurrent branch of the anterior interosseous artery $6.0 \mathrm{~cm}$ proximal to the ulnar head was detected preoperatively using CT angiography and handheld Doppler (Figure 4). The recurrent branch of anterior interosseous artery perforator-based propeller flap was designed adjacent to the previous incision (Figure 3D). A healthy septocutaneous perforator emerged through the septum between the extensor digiti minimi and extensor carpi ulnaris (Figure 3E). The flap was raised in the same manner as in the previous case and was rotated by $120^{\circ}$ clockwise to cover the defect (Figure 3F). The size of the flap was $21 \mathrm{~cm} \times 4 \mathrm{~cm}$. The donor site was closed using a free skin graft. Postoperative rehabilitation was started at 7 days after reconstruction. After the start of rehabilitation, the patient experienced hemarthrosis in the third MCP joint, which rapidly healed without infection after evacuation via a small incision. There were no other complications, and the flap completely survived. The patient was referred to the rehabilitation department at 1 month postoperatively and returned to daily activities at 4-month follow-up (Figure 3G). 


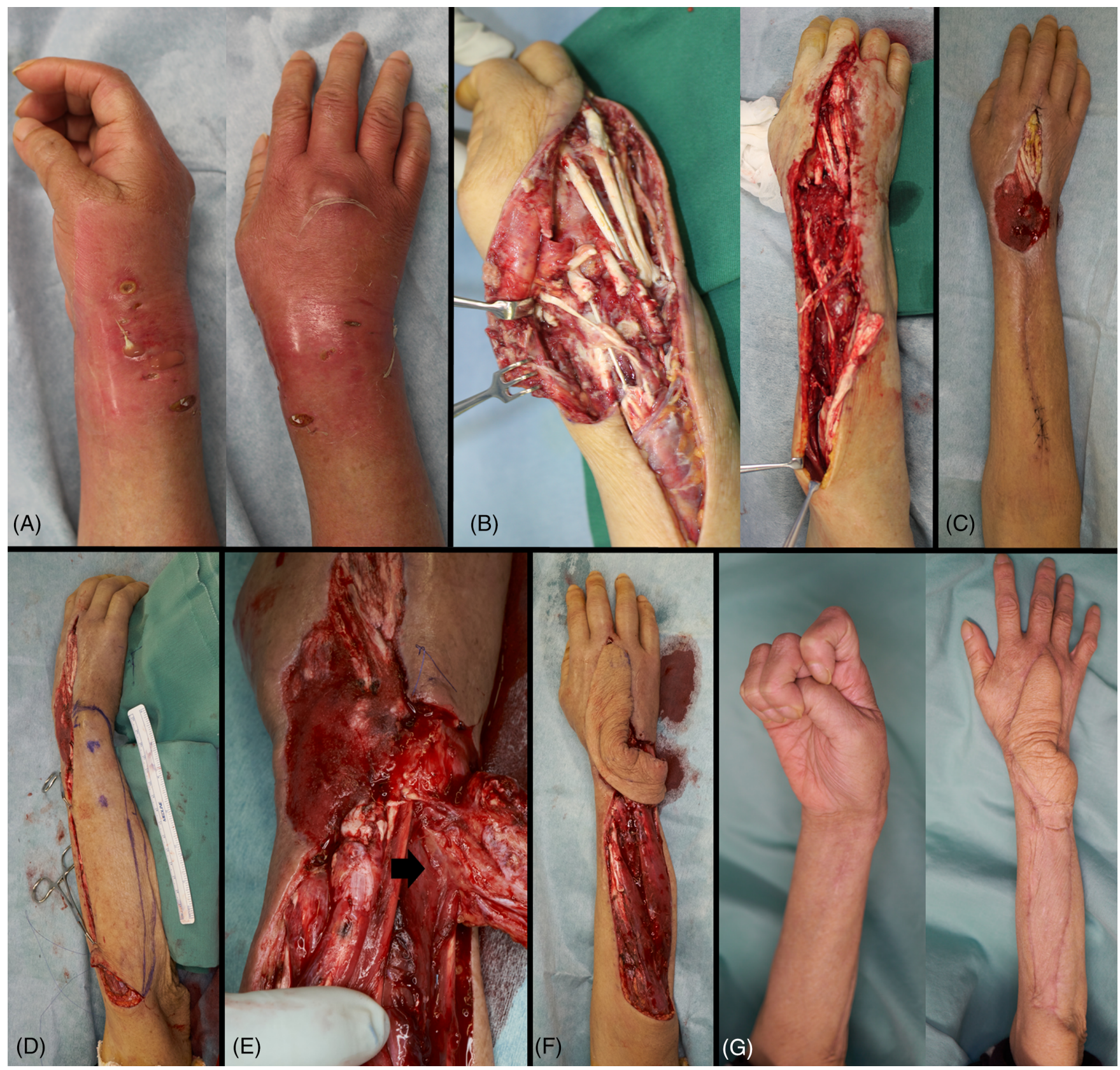

FIGURE 3 A, Wound on the right distal forearm. B, Degenerated tendons and intraoperative view of debridement. C, After performance of repeated debridement. D, Flap design. E, Recurrent branch of the anterior interosseous artery perforator (black arrow). $\mathrm{F}, \mathrm{After} 120^{\circ}$ of rotation. G, At 6 months after surgery

\section{3 | DISCUSSION}

In this paper, we presented our experience with 2 cases treated with the recurrent branch of anterior interosseous artery perforator-based propeller flap. Numerous similar perforator-based flaps in the distal forearm have been well described in the published literature. The anterior interosseous artery perforator-based flap is a well-known flap for distal forearm reconstruction (Akin, 2003; Panse et al., 2017; Panse \& Sahasrabudhe, 2014; Syed et al., 1997; Yii \& Niranjan, 1999). However, to the best of our knowledge, there has been no report describing the recurrent branch of anterior interosseous artery perforator-based propeller flap for distal forearm defects. In the case of reverse posterior interosseous artery flaps, additional procedures with wide distal flap base including perforating branches from the anterior or posterior interosseous artery have been reported to enhance the reliability of vascular supply (Chen et al., 1998; Giunta \& Lukas, 1998). In our cases, the flaps based on only 1 perforator extended to two-thirds or almost the full length of the forearm from the recurrent branch of the anterior interosseous artery without including other perforators from the posterior interosseous artery, and it survived completely. Although there is no definitive information about the survival area of this flap, the anatomical relationship between the recurrent branch of the anterior interosseous artery and the posterior interosseous artery has been well described in previous reports in the literature (Angrigiani, Grilli, Dominikow, \& Zancolli, 1993; Hubmer et al., 2004; Sun et al., 2015). In an extensive cadaveric 


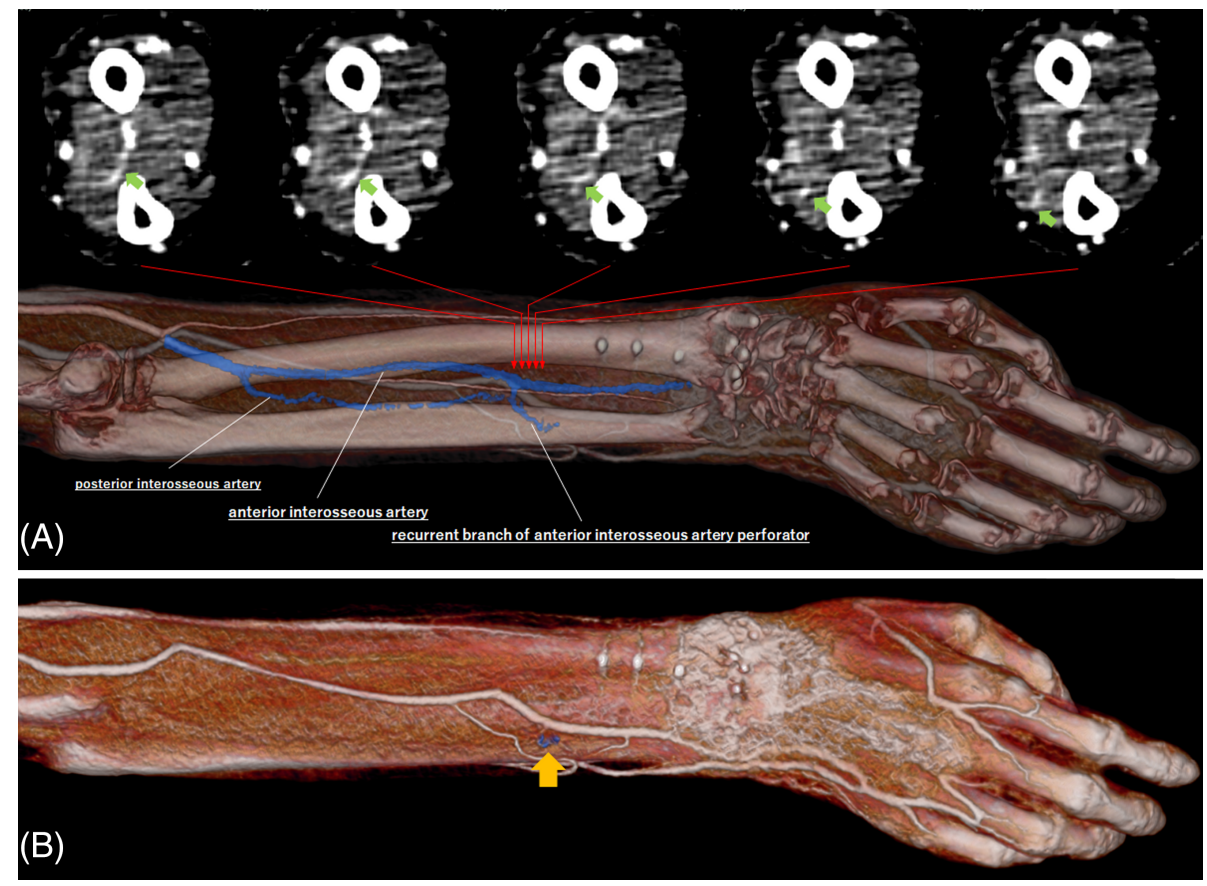

FIGURE 4 Multidetector computed tomographic angiography (MDCTA) and 3D fusion images of the interosseous vascular system (blue vessel) in case 2. A, Axial views of the MDCTA at each level (red arrows) and the recurrent branch of the anterior interosseous artery perforator (green arrows). B, Emerging point of the perforator through the septum between the extensor digiti minimi and extensor carpi ulnaris (yellow arrow)

study, Humber et al. performed anatomic and embryologic analysis of the anastomosis between the anterior and posterior interosseous arteries and, based on the diameter of the posterior interosseous artery, concluded that the vascular arcade formed by the dorsal branch of the anterior interosseous artery anastomoses with the posterior interosseous artery via choke vessels in the middle forearm. Furthermore, they proposed the term "recurrent branch of the anterior interosseous artery" to describe the distal portion of the posterior interosseous artery (Hubmer et al., 2004). Sun et al. performed a statistical analysis of the perforator location of the posterior interosseous vascular system. They reported that 2 main clusters of perforators from the posterior interosseous vascular system were located at a distance of $6 \pm 2 \mathrm{~cm}$ proximal to the head of the ulna and $10 \pm 1 \mathrm{~cm}$ distal to the lateral epicondyle of the humerus along the axis of the ulnar head-to-lateral epicondyle (Sun et al., 2015). These reports indicate that the cluster of perforators proximal to the head of the ulna is derived from the anterior interosseous vascular system, whereas the cluster of perforators distal to the lateral epicondyle of the humerus is derived from the posterior interosseous vascular system. Consequently, they connect to each other via choke anastomosis in the middle forearm. Therefore, the recurrent branch of anterior interosseous artery perforator-based flap, including additional adjacent perforator vascular territories of the posterior interosseous artery, may be harvested at a large size to provide sufficient coverage for the distal forearm in accordance with the perforasome theory (Taylor, Chubb, \& Ashton, 2013; Taylor, Corlett, \& Ashton, 2017). Angrigiani et al. performed ink injections in fresh cadaver forearms through a catheter placed in the distal portion of the anterior interosseous artery. In their study, the distal and middle thirds of the dorsal forearm were stained, but the proximal third remained unstained despite the larger amounts of ink injected. Further, they observed that tissue loss occurred in the reverse posterior interosseous artery flap when raised in the proximal third of the forearm despite the incorporation of the large proximal cutaneous branch of the posterior interosseous artery in the pedicle (Angrigiani et al., 1993). According to their studies, harvesting the recurrent branch of anterior interosseous artery perforator-based propeller flap extending to two-thirds of the dorsal forearm may be safe; however, reaching a defect extending beyond the MCP joint seems difficult. The rotation arc of the skin paddle is limited by the location of the perforators. Therefore, preoperative assessment of vascular information by handheld Doppler or CT angiography is required. Although the location of the perforators can be predicted using preoperative handheld Doppler or CT angiography, the surviving area of the recurrent branch of anterior interosseous artery perforator-based flap remains uncertain. We encountered only 2 cases, and further study is required to clarify the vascular territory of this flap.

Although the reverse posterior interosseous artery flap is a "workhorse" for distal forearm reconstruction, it has disadvantages in that impossible cases of flap exist in minority of patients with anatomical blood vessel variation (Cavadas, 1999; Dadalt Filho et al., 1994; Giunta \& Lukas, 1998). Therefore, it is important to consider an alternative procedure when planning for the reverse posterior interosseous artery flap. However, there are few salvage procedures for the impossible case of reverse posterior interosseous artery flap. Giunta et al. and Pauchot et al. described a case with absent pedicle of the posterior interosseous artery flap and the salvage procedure in this situation (Giunta \& Lukas, 1998; Pauchot et al., 2010). However, their solutions require complex procedures such as conversion to a free flap or anterior interosseous artery flap with dissection of the interosseous membrane. As described above, a situation in which there are no standardized salvage techniques for the impossible case of reverse 
posterior interosseous artery flap is challenging. In our experience with 1 case, the procedure could be changed from the reverse posterior interosseous artery flap to the recurrent branch of anterior interosseous artery perforator-based propeller flap via an existing incision. The recurrent branch of anterior interosseous artery perforator-based propeller flap has duplicative vascular territory of the posterior interosseous artery flap. Therefore, it can archive only additional incision continuing the previous incision and superficial dissection around the pedicle perforator. In our case, the recurrent branch of anterior interosseous artery perforator-based propeller flap could serve as a substitute for the reverse posterior interosseous artery flap, hence providing a simple local solution.

The anterior interosseous vascular system passes between the ulna and radius adjacent to the interosseous membrane, which may reduce the probability of damage to the vascular pedicle of the recurrent branch of anterior interosseous artery perforator-based flap. The vascular supply of the recurrent branch of anterior interosseous artery perforator-based propeller flap seems stable owing to the provision of double arterial blood supply from the anterior and posterior interosseous arteries with antegrade venous drainage. Further, a compensatory increase in anterior and posterior interosseous artery perfusion was noted after radial artery excision (Ciria-Lloréns, Gómez-Cía, \& Talegón-Meléndez, 1999), which may be beneficial to patients with ulnar or radial artery injury. However, if there is suspected disruption of the anterior interosseous artery, impairment of flap circulation could be possible, and other procedures should be considered.

Donor site morbidity and cosmetic consideration are also important when planning distal forearm reconstruction. Neuwirth et al. evaluated the donor site morbidity of posterior interosseous artery flaps and reported high satisfaction with the aesthetic appearance of donor sites; however, there was a difference between directly closed and grafted donor sites, with acceptance and satisfaction being lower for grafted donor sites (Neuwirth, Hubmer, \& Koch, 2013). The recurrent branch of anterior interosseous artery perforator-based propeller flap leaves a visible scar at the donor site, and it may require free skin graft, which may be unacceptable, especially in young female patients.

We described 2 cases of the recurrent branch of anterior interosseous artery perforator-based propeller flap. In 1 case, the reverse posterior interosseous artery flap could be converted to the recurrent branch of anterior interosseous artery perforator-based propeller flap. We believe that it is a reasonable option for coverage of the distal forearm, especially in impossible cases of reverse posterior interosseous artery flap.

\section{ORCID}

Ryusho Hamada (D) https://orcid.org/0000-0002-4035-823X

\section{REFERENCES}

Akin, S. (2003). V-Y advancement island flap based on the perforator of the anterior interosseous artery. Annals of Plastic Surgery, 51, 51-56.

Akinci, M., Ay, S., Kamiloglu, S., \& Erçetin, O. (2006). The reverse posterior interosseous flap: A solution for flap necrosis based on a review of
87 cases. Journal of Plastic, Reconstructive \& Aesthetic Surgery, 59 148-152.

Angrigiani, C., Grilli, D., Dominikow, D., \& Zancolli, E. A. (1993). Posterior interosseous reverse forearm flap: Experience with 80 consecutive cases. Plastic and Reconstructive Surgery, 92, 285-293.

Biemer, E., \& Stock, W. (1983). Total thumb reconstruction: A one-stage reconstruction using an osteo-cutaneous forearm flap. British Journal of Plastic Surgery, 36, 52-55.

Buchler, U., \& Frey, H. P. (1991). Retrograde posterior interosseous flap. Journal of Hand Surgery, 16, 283-292.

Cavadas, P. C. (1999). Posterior interosseous flap based on a septal perforator, in the absence of the distal artery. Plastic and Reconstructive Surgery, 104, 592.

Chen, H. C., Cheng, M. H., Schneeberger, A. G., Cheng, T. J., Wei, F. C., \& Tang, Y. B. (1998). Posterior interosseous flap and its variations for coverage of hand wounds. The Journal of Trauma, 45, 570-574.

Ciria-Lloréns, G., Gómez-Cía, T., \& Talegón-Meléndez, A. (1999). Analysis of flow changes in forearm arteries after raising the radial forearm flap: A prospective study using colour duplex imaging. British Journal of Plastic Surgery, 52, 440-444.

Dadalt Filho, L. G., Ulson, H. J., \& Penteado, C. V. (1994). Absence of the anastomosis between the anterior and posterior interosseous arteries in a posterior interosseous flap: A case report. Journal of Hand Surgery, 19, 22-25.

Giunta, R., \& Lukas, B. (1998). Impossible harvest of the posterior interosseous artery flap: A report of an individualised salvage procedure. British Journal of Plastic Surgery, 51, 642-645.

Hubmer, M. G., Fasching, T., Haas, F., Koch, H., Schwarzl, F., Weiglein, A., \& Scharnagl, E. (2004). The posterior interosseous artery in the distal part of the forearm. Is the term "recurrent branch of the anterior interosseous artery" justified? British Journal of Plastic Surgery, 57, 638-644.

Hyakusoku, H., Yamamoto, T., \& Fumiiri, M. (1991). The propeller flap method. British Journal of Plastic Surgery, 44, 53-54.

Jones, B. M., \& O'Brien, C. J. (1985). Acute ischaemia of the hand resulting from elevation of a radial forearm flap. British Journal of Plastic Surgery, 38, 396-397.

Li, Z. T., Liu, K., \& Cao, Y. D. (1989). The reverse flow ulnar artery island flap: 42 clinical cases. British Journal of Plastic Surgery, 42, 256-259.

Neuwirth, M., Hubmer, M., \& Koch, H. (2013). The posterior interosseous artery flap: Clinical results with special emphasis on donor site morbidity. Journal of Plastic, Reconstructive \& Aesthetic Surgery, 66, 623-628.

Özalp, B., Elbey, H., Aydin, A., \& Özkan, T. (2016). Distally based subcutaneous veins for venous insufficiency of the reverse posterior interosseous artery flap. Microsurgery, 36, 384-390.

Panse, N., \& Sahasrabudhe, P. (2014). Free style perforator based propeller flaps: Simple solutions for upper extremity reconstruction! Indian Journal of Plastic Surgery, 47, 77-84.

Panse, N. S., Joshi, S. B., Sahasrabudhe, P. B., Bahetee, B., Gurude, P., \& Chandanwale, A. (2017). The anterior interosseus artery perforator flap: Anatomical dissections and clinical study. World Journal of Plastic Surgery, 6, 152-158.

Pauchot, J., Lepage, D., Leclerc, G., Flamans, B., Obert, L., \& Tropet, Y. (2010). Posterior interosseous free flap because of absence of posterior interossous pedicle. A report of an individualised salvage procedure and of an exceptional anatomical variation. Review of the literature. Annales de Chirurgie Plastique et Esthétique, 55, 56-60.

Shaw, D. T., \& Payne, R. L., Jr. (1946). One stage tubed abdominal flaps; Single pedicle tubes. Surgery, Gynecology \& Obstetrics, 83, 205-209.

Sun, C., Wang, Y. L., Ding, Z. H., Liu, P., Qin, X. Z., Lee, H. L., \& Jin, A. M. (2015). Anatomical basis of a proximal fasciocutaneous extension of the distal-based posterior interosseous flap that allows exclusion of the proximal posterior interosseous artery. Journal of Plastic, Reconstructive \& Aesthetic Surgery, 68, 17-25.

Syed, S. A., Zahir, K. S., Zink, J. R., Saglaam, O., Restifo, R. J., \& Thomson, J. G. (1997). Distal dorsal forearm flap. Annals of Plastic Surgery, 38, 396-403.

Taylor, G. I., Chubb, D. P., \& Ashton, M. W. (2013). True and "choke" anastomoses between perforator angiosomes: Part I. anatomical location. Plastic and Reconstructive Surgery, 132, 1447-1456. 
Taylor, G. I., Corlett, R. J., \& Ashton, M. W. (2017). The functional angiosome: Clinical implications of the anatomical concept. Plastic and Reconstructive Surgery, 140, 721-733.

Yii, N. W., \& Niranjan, N. S. (1999). Fascial flaps based on perforators for reconstruction of defects in the distal forearm. British Journal of Plastic Surgery, 52, 534-540.

Zancolli, E. A., \& Angrigiani, C. (1986). Colgajo dorsal de antebrazo (en isla). La Revista de la Asociación Argentina de Ortopedia y Traumatología, 51, 161-168.
How to cite this article: Hamada R, Shinaoka A, Sugiyama N, Watanabe T, Miura Y, Kimata Y. Recurrent branch of anterior interosseous artery perforator-based propeller flap for distal forearm injuries: Report of 2 cases. Microsurgery. 2018;38:

917-923. https://doi.org/10.1002/micr.30388 